\title{
Computational Steering of Complex Flow Simulations
}

\author{
Atanas Atanasov ${ }^{1}$, Hans-Joachim Bungartz ${ }^{1}$, Jérôme Frisch ${ }^{2}$, Miriam Mehl ${ }^{1}$, \\ Ralf-Peter Mundani ${ }^{2}$, Ernst Rank ${ }^{2}$, and Christoph van Treeck ${ }^{3}$
}

\begin{abstract}
Computational Steering, the combination of a simulation back-end with a visualisation front-end, offers great possibilities to exploit and optimise scenarios in engineering applications. Due to its interactivity, it requires fast grid generation, simulation, and visualisation and, therefore, mostly has to rely on coarse and inaccurate simulations typically performed on rather small interactive computing facilities and not on much more powerful high-performance computing architectures operated in batch-mode. This paper presents a steering environment that intends to bring these two worlds - the interactive and the classical HPC world - together in an integrated way. The environment consists of efficient fluid dynamics simulation codes and a steering and visualisation framework providing a user interface, communication methods for distributed steering, and parallel visualisation tools. The gap between steering and HPC is bridged by a hierarchical approach that performs fast interactive simulations for many scenario variants increasing the accuracy via hierarchical refinements in dependence of the time the user wants to wait. Finally, the user can trigger large simulations for selected setups on an HPC architecture exploiting the pre-computations already done on the interactive system.
\end{abstract}

\section{Introduction}

Computational Steering is the coupling of a simulation back-end with a visualisation front-end in order to interactively exploit design alternatives and/or optimise (material) parameters and shape. Therefore, different aspects such as grid generation, efficient algorithms and data structures, code optimisation, and parallel computing play a dominant role to provide quick results (i. e. several simulation and visuali-

Department of Informatics, Technische Universität München e-mail: \{atanasoa,bungartz,mehl\}@in.tum.de . Chair for Computation in Engineering, Technische Universität München e-mail: \{frisch,mundani,rank\}@bv.tum.de · Fraunhofer-Institut für Bauphysik, Holzkirchen e-mail: treeck@ibp.fraunhofer.de

This is a pre-print of an article published in Wagner S., Steinmetz M., Bode A., Müller M. (eds) High Performance Computing in Science and Engineering, Garching/Munich 2009. The final authenticated version is available online at: https://doi.org/10.1007/978-3-642-13872-0_6 
sation updates per second in case of modifications of the underlying data) to keep up the principle of cause and effect, which is necessary to gain better insight and a deeper understanding of problems from the field of engineering applications. Nevertheless, even nowadays interactivity and high-performance computing (HPC) are still a contradiction, as most HPC systems do not provide interactive access to the hardware.

As a remedy for the latter one, a two-stage approach (i.e. interactive preprocessing of "low level" problems and parallel processing of "high level" problems) helps to bridge the gap between small - and typically interactive - systems for a quick quantitative analysis and large - and typically batch - HPC systems for a complex qualitative analysis. Such an approach also provides the advantage of reducing the amount of long and, thus, expensive simulation runs to those necessary only without waisting additional computing time for redundant computations. To ensure a seamless transition from "low level" problems on coarse grids with few thousands of unknowns to "high level" problems on fine grids with many millions of unknowns, hierarchical approaches are indispensable.

This also has a significant relevance for the practical usage of computational steering and HPC in industrial applications, as most approaches there suffer from a insufficient integration of HPC into the workflow of industrial processes. Hence, from the very beginning one of our main objectives was to provide a framework for engineering applications that not only addresses challenging mathematical and computer science related questions, but also combines and consolidates the two conflicting aspects of interactivity and high-performance computing. Therefore, we will show the benefits of our framework for the interactive control of different engineering applications running on parallel architectures.

The remainder of this paper is as follows. Section 2 presents the ingredients of the steering environment. As this environment does not cover the whole range of applicability of the underlying approaches, Sect. 3 describes two further applications that have been or will be coupled to the steering and visualisation framework. Finally, we draw a short conclusion and give an outlook on the future work in Sect. 4.

\section{Computational Steering Environment}

In order to increase the performance, i. e. decrease simulation and visualisation response time of our steering environment as well as to prepare it for a later HPC usage, several measures have been taken. This was done with a straight focus on the two-way approach as described above, where small systems are used for an interactive data exploration before a high-quality analysis (based on the parameters explored) is launched as (massively) parallel job on large HPC systems. 


\subsection{Hierarchical Approach}

The main idea in joining the interactively computed small systems with the large parallel systems computed on HPC architectures is to exploit hierarchies of grid levels or discretisation orders. As a response on each user input, a simulation on a very coarse grid or with lowest discretisation order is triggered such that first visualised results are available very fast. Depending on the time given - that is the time the user wants to wait for more accurate results - the simulation is refined in a recursive manner. Each of these refinement steps adds a new layer of grid points to decrease the mesh width or additional degrees of freedom at existing grid points to enhance the approximation order. This allows to quickly check results for numerous input configurations, to examine those that seem to be relevant more accurately and, finally, to start large HPC simulations only for a few scenarios of particular interest. Hereby the refined simulations already profit from the coarser ones in a full multigrid manner. Codes such as iFluids, Peano, and the $p$-FEM structural mechanics codes described below naturally fit this approach as they inherently already provide the required hierarchy.

\section{2 iFluids}

The kernel of our steering framework is a Lattice-Boltzmann fluid solver which has been developed by our group and ported to the former HPC system - the pseudovector computer Hitachi SR8000-F1 - installed at Leibniz-Rechenzentrum (LRZ). This fluid solver - called iFluids [15] - was running interactively on the Hitachi while coupled with the interactive visualisation nodes also available at LRZ for computational steering applications. Due to the replacement of the old HPC system with the SGI Altix 4700 severe changes of iFluids became necessary in order to run it successfully on the new system. These changes comprise to switch from a pure MPI-based implementation to a cache-efficient hybrid approach (MPI/OpenMP) to benefit also from the Itanium CPUs' local shared memory as well as to modifiy the communication and data distribution pattern, such that it optimally suits the underlying network topology ( $2 \mathrm{D}$ tori connected via a fat tree) in order to minimise latency.

As the porting of iFluids is still work in progress, current performance measurements (up to 1024 processes) on the Altix do not yet reveal the full potential of the parallel code, nevertheless already sound very promising. For a problem size with 7.5 million degrees of freedom a nearly linear speedup (strong scaling) up to $p=64$ processes could be observed which strongly drops for growing numbers of $p$ (see Fig. 1).

Further investigations on this behaviour showed that the major drawback of the current parallelisation is the regular block decomposition of the domain that leads to partitions consisting of mostly or entirely obstacle cells only, for which no computation have to be performed. This leads to an unbalanced load situation. Therefore and 

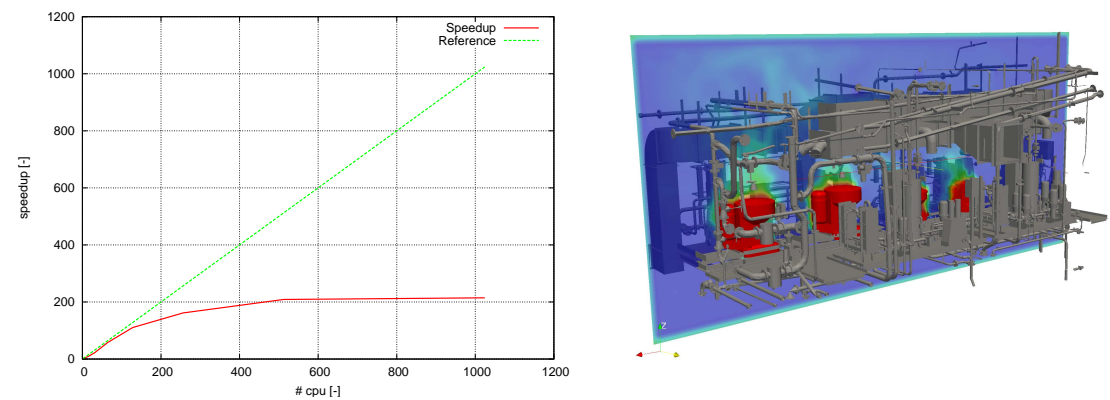

Fig. 1 Performance results running iFluids on the SGI Altix 4700 - left-hand side shows strong speedup values, right-hand side shows simulation results for a complex geometry.

due to the frequent geometry and refinement depth changes in a steering environment, a more enhanced adaptive and dynamical load balancing strategy is inevitable. A modified master-slave concept which has been developed by our group (see next section) is being incorporated into iFluids at the moment.

\subsection{Adaptive Load Balancing}

Within a related project for structural analysis using the $p$-version finite element method ( $p$-FEM, [12]) - i. e. increasing the polynomial degree $p$ of the shape functions for better accuracy without changing the discretisation - a similar behaviour regarding unbalanced load situations has been observed when using a hierarchical approach (octrees) for domain decomposition [8]. Therefore, we have implemented an adaptive load balancing strategy based on the idea of task stealing-a modified master-slave concept, that takes into account varying workload on the grid nodes.

Here, a master process first analyses the tree and estimates the total amount of work (measured in floating-point operations) per node. In the next step, those nodes are assigned to processes called traders - an intermediate layer between master and slaves - to prevent communication bottlenecks in the master and, thus, making this approach also scaleable for large amounts of processes. The traders define tasks (i. e. systems of linear equations for domain partitions), "advertise" them via the master to the slaves, and take care about the corresponding data transfer. They also keep track about dependencies between the tasks and update those dependencies with each result sent back from a slave. Benchmark computations with different ratios of traders and slaves have shown good results with respect to the average percentage a single slave is busy during the entire runtime. This is important to obtain high update rates in case of frequent re-computations which are necessary for interactive computational steering applications.

Hence, iFluids can also benefit from this approach. By applying a hierarchical organisation of the computational domain, the master process could easily identify re- 
gions mostly consisting of obstacle cells when doing its work load estimation. Such a region could then be combined with neighbouring regions to a larger task which is processed by a single slave to achieve a better computation-communication-ratio. As this is still work in progress, there are no current results so far.

\subsection{Remote Visualisation and Steering Framework}

For fast visualisation and user interaction, a remote and parallel visualisation and steering framework has been developed in [2]. It is based on the idea of a distributed application. That is, the steering and visualisation application, the underlying simulation, and the user interface run on separate computing facilties. The interaction between these components is realised via remote procedure calls (RCP) and TCP sockets. As our task is to bring together interactive simulations and visualisations with HPC applications, i. e. large systems of equations to be solved and large data sets to be visualised, the visualisation and simulation are parallel processes themselves as displayed in Fig. 2.

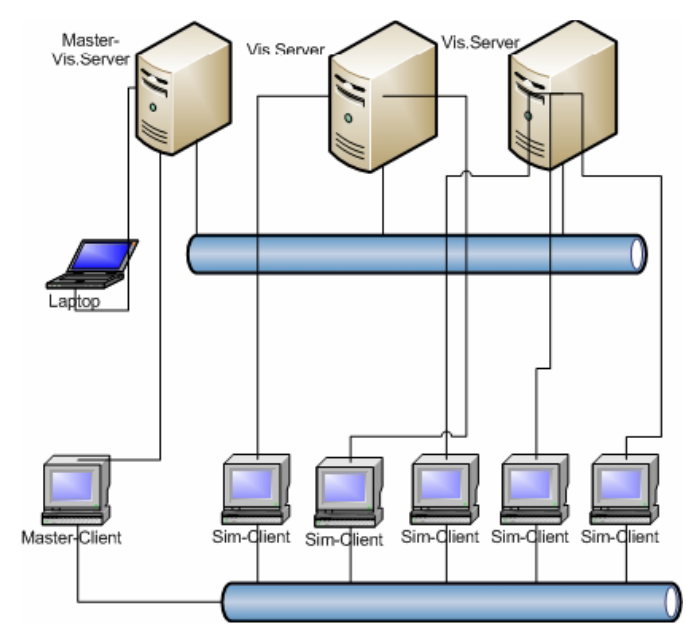

Fig. 2 Steering environment with parallel remote visualisation and parallel simulation (taken from [2]).

The visualisation is based on the Visualization Toolkit (VTK, [10, 7]). For scalar data sets, it provides a colour mapping as well as iso-lines or iso-surfaces enhanced by cutting planes that can be displaced and rotated interactively. Vector data such as flow velocities are visualised using streamlines, dashed streamlines with glyphs, or 
streambands. Geometries are represented by surface triangulations and a bounding box widget that allows to scale, displace, or rotate the geometry.

The user interface consists of a 3D-viewer, a geometry catalogue, a geometry browser, and a control panel. It allows the user to change geometries (add, delete, move, or scale geometrical objects), choose data to be visualised (velocities or pressure, e. g.), select visualisation techniques (streamlines or streambands, e. g.), and to examine simulation results from different views and with different techniques. Figure 3 shows a screenshot of the user interface with a visualisation of a fluid dynamics scenario.

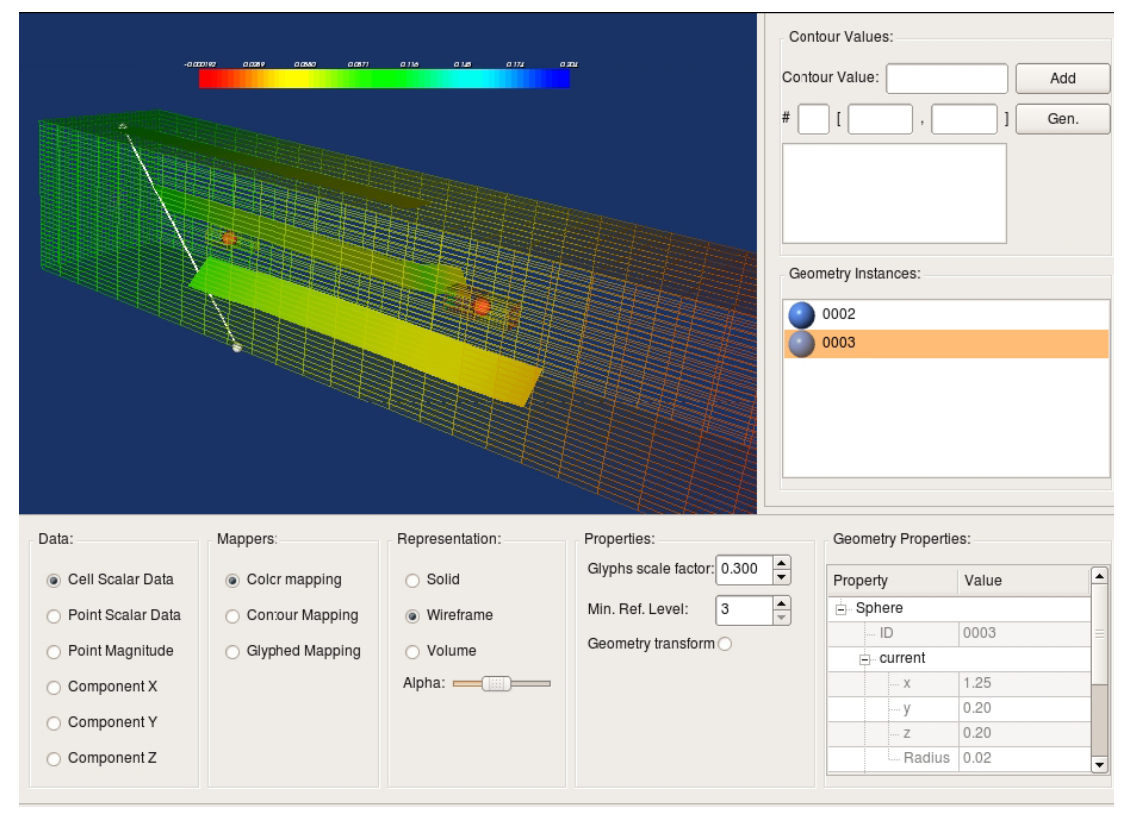

Fig. 3 Steering User Interface with a streamband visualisation for a flow computed with the Peano CFD solver. The scenario is a channel flow with a spherical obstacle. The second sphere has been added at runtime. At the right bottom, properties of the geometry are displayed (taken from [2]).

The visualisation is parallelised following a data parallel approach. Visualisations are performed in parallel for subdomains of the entire scenario. The bottleneck of this approach is the composition of all subdomain pictures to a picture of the entire scenario at the end of the visualisation process. A binary space partition (BSP) tree approach avoids the accumulation of the whole composition work in one master process. It recursively joins pictures associated to the same father in a bottom-up traversal of the BSP tree.

Figure 4 shows an example of a domain splitting using a BSP tree. In this example, the subdomains $D$ and $E$ would be joined first to a larger domain $D E$. In 
a second step, $A$ and $B$ would be joined to $A B$. In parallel, $D E$ would be joined with $C$ to $C D E$, and, finally, $A B$ and $C D E$ would be joined to the entire scenario. In our applications such as Peano, we use a particular form of BSP trees - octree-like space-partitioning trees.

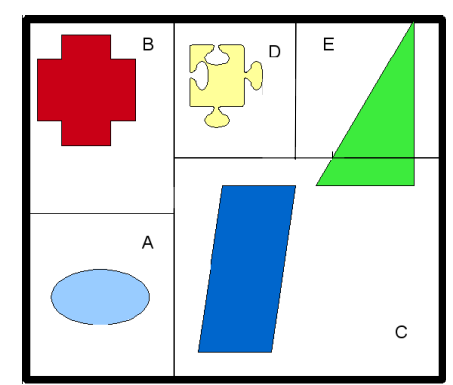

(a)

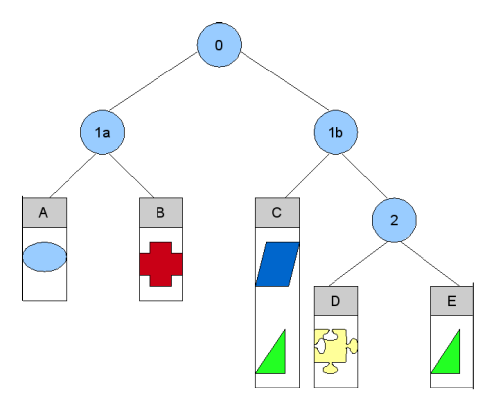

(b)

Fig. 4 Example for a BSP-tree domain partitioning for parallel visualisation (taken from [2]). (a) Spatial decomposition according to the BSP-tree; (b) BSP-tree and data structure for this example.

In case of Peano (see Sect. 3.1) as simulation code, it is not neccessary to define a new BSP-tree decomposition of the domain for visualisation purposes as Peano already provides it for its own domain decomposition. As this decomposition is already done in a load balanced way and, in case of a non- $p$-adaptive code such as Peano, simulation costs as well as visualisation costs per inner domain node are approximately constant, it can be efficiently used also for parallel visualisation. Test runs with the steering framework and the CFD solver Peano have been performed at the Linux Cluster (eight-way AMD Opteron, $2.6 \mathrm{GHz}, 32$ GByte RAM per node) at Leibniz Supercomputing Center (LRZ) in Garching. The visualisation has been done on a Sun X4600 Server with eight quad-core Opterons with 256 GByte RAM per processor and four Nvidia Quadro FX5800 graphic cards. Figure 5 shows the resulting speedup and the costs for picture composition. These results are preliminary and still offer a wide range of optimisation properties both in terms of the number of processors used and in terms of the speedup.

\section{Related Applications}

In the following, we will highlight some related applications that have been developed independent from iFluids. The first one, the Navier-Stokes solver of the framework Peano, has been the test application during the development of the steering framework. The second one, a thermal comfort assessment application, is a steering application not yet directly related to high-performance computing. However, to refine the underlying model - which will be neccessary in the future - also fluid 


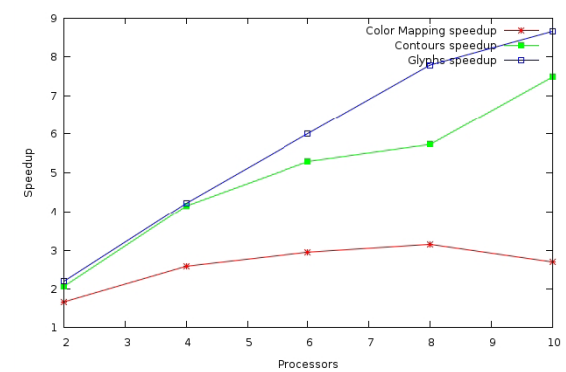

(a)

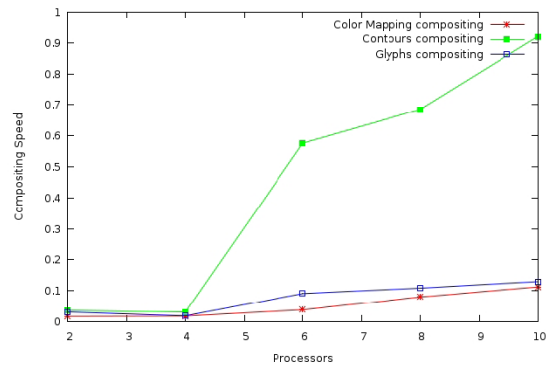

(b)

Fig. 5 Speedup evaluation of the parallel visulisation with a domain partitioning defined by the Peano solver and costs for the visualisation composition (taken from [2]). (a) Speedup of the colour mapping, contour generation, and glyphing. (b) Runtime for the composition of pictures.

dynamics will have to be included in the model which will than strongly be related to the main focus of this paper.

\subsection{Peano}

Peano is a solver framework for partial differential equations (PDE) that works on adaptively refined Cartesian grids corresponding to octree-like tree structures, socalled space-partitioning grids [16]. Within this framework, a Navier-Stokes solver with dynamical grid refinement is implemented [9]. This code fits perfectly with the steering concept described above as it naturally provides the grid hierarchy required for the hierarchical integration of interactive simulations with large HPC batch jobs for selected scenarios. Figure 6 (a) shows the grid hierarchy for a simple two-dimensional example.

The unique selling points of Peano are low memory requirements in combination with high cache hit-rates, efficient multiscale solvers, and efficient and parallel tree-based domain decomposition. Peano has been run on the HLRB II at the Leibniz Supercomputing Center in Garching on up to 900 processors with a speedup of 700 [4]. It can handle moving objects leading to arbitrarily large geometry or even topology changes as it is based on a fixed (Eulerian) grid. Only the adaptive grid refinement is adjusted according a deforming, moving, deleted, or added object (see Fig. 6 (b)). Such, also particles advected in a flow field can be simulated in a very efficient way [3].

Due to its suitability for both the hierarchical integration approach and the parallel tree-based domain decomposition that can also be used for parallel visualisation, the test runs for the steering framework described in the previous section, have been performed with Peano as a simulation code. 


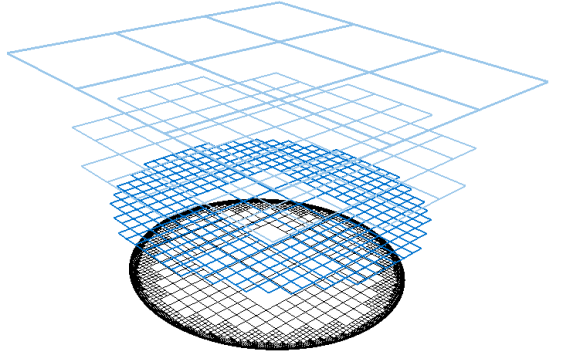

(a)
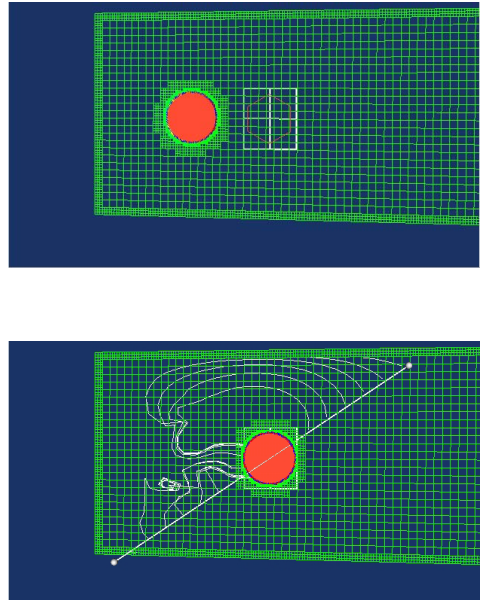

(b)

Fig. 6 Peano grids for two-dimensional examples. (a) Grid hierarchy for a spherical domain; (b) adaptive grid refinement following a moving shpere (taken from [2]).

\subsection{Thermal Comfort Assessment}

\subsubsection{Motivation}

Indoor climate predictions in office buildings gained increasing importance in the past. The aim of reducing the energy consumption of buildings, and maintaining reasonable indoor temperatures for the occupants at the same time, can be accomplished using simulation tools in the early design stages of the design phase.

In the broader context of the underlying research project COMFSIM [15] three modules were defined. In a first study, a virtual climate chamber [14] was designed, which makes use of a human thermoregulation model according to Fiala [5]. Occupants can be situated in a rectangular enclosure with well-defined boundary conditions, such as room and surface temperatures, relative humidity, air velocity and metabolic rate. The latter quantities can be changed during an ongoing simulation using the computational steering concept.

The numerical thermal manikin can be coupled with iFluids [15]. After a series of iterations of the CFD solver, the current boundary conditions at the surface of the manikin shall be delivered to the thermoregulation interface. The existing interface provides the thermal state of the manikin in terms of the resultant surface temperatures and heat fluxes, which may act as new boundary conditions of the manikin in the next CFD step. Using these resulting surface temperatures, a local comfort vote can be calculated using a 7 point ASHRAE scale [1], for example, indicating the comfort state of the manikin. The developed local assessment method of our postprocessing tool has already been published by the authors in [14]. Coupling CFD 
with the numerical manikin offers the possibility to predict the indoor thermal comfort situation in detail, such as assessing the draught risk, asymmetric radiation, etc. [13]

\subsubsection{Thermoregulation Modeling}

Thermoregulatory reactions of the central nervous system are an answer of multiple functions of signals from core and peripherals. Local changes in skin temperature additionally cause local reactions such as modifying the sweating rate or the local vasodilatation. Significant indicators are the mean skin temperature and its variation over time and the hypothalamus temperature. The indicators can be correlated with the autonomic responses in order to form a detailed thermoregulation model $[5,11]$.

Detailed manikin models usually consist of a passive system dealing with physical and physiological properties, including the blood circulation and an active thermoregulation system for the afferent signals analysis [11]. Local clothing parameters are taken into account and the response of the metabolism can be simulated over a wide range of ambient conditions. Besides two-node models (Gagge) [6], multisegment models are known which are founded on the early work of Stolwijk [11]. Most models use a decomposition of the human body into layers and segments for the passive system which are in thermodynamic contact with each other and with the ambient environment.

As mentioned in section above, the numerical approach for the evaluation of the human thermoregulation for this application was chosen to be the Fiala model. Detailed information can be found in [5].

\subsubsection{Computational Steering Approach}

Fig. 7 Coupling concept: virtual climate chamber in computational steering mode

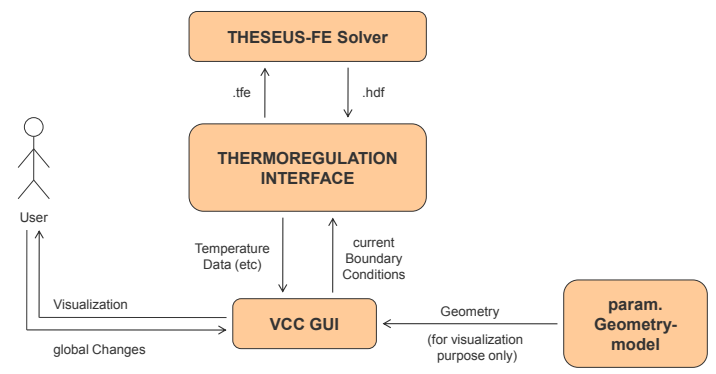

The above mentioned procedure can be embedded in a computational steering context. Figure 7 shows the coupling of the virtual climate chamber (VCC) with the thermoregulation interface. The user loads the geometry in to the virtual climate 
chamber for visualization. There global boundary conditions can be set, governing the chamber climate. The data is transfered to the thermoregulation interface which is coupled to a numerical solver. The aim of the interface is to provide standard interface functions in a way that the numerical model could be exchanged easily. The numerical model computes a small timestep and delivers the results to the interface which sends them to the virtual climate chamber for visualisation purposes. Depending on the just shown results, the user might want to alter some of the boundary conditions which will be again transfered to the interface for further treatment and so on.

Fig. 8 Coupling concept: external CFD solvers or zonal models coupled with the thermoregulation simulation

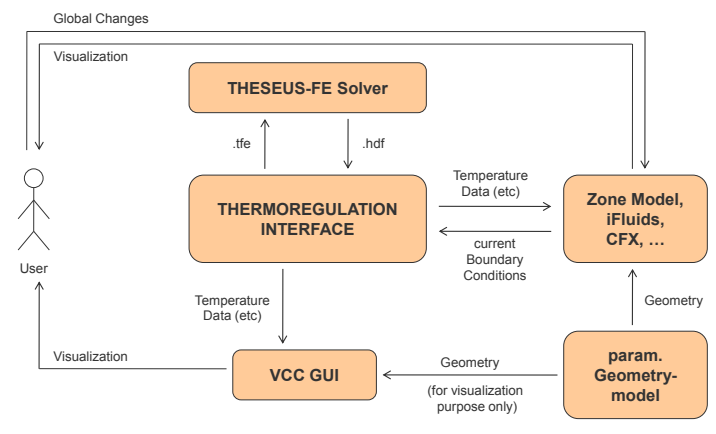

This procedure is nice for test cases, but is hardly applicable in real applications. Therefore a more realistic coupling is depicted in Fig. 8. The user starts a CFD computation which loads the geometry and scene information. Manikins are now embedded in the geometry and classified as thermal active components. The CFD code computes a fixed amount of timestep and delivers the local velocities and temperatures at the manikin's surface which will be transfered to the thermoregulation interface who will pass them on to the solver and deliver the results back to the interface. The resultant surface temperatures are given to the CFD computation which will act as new boundary conditions in the next CFD step. The virtual climate chamber is connected to the thermoregulation interface in view only mode in order to observe further detailed information about the numerical thermoregulation simulation like mean values for the whole body as mean skin temperature etc.

\section{Summary and Outlook}

We proposed tools that combine efficient HPC flow solvers with a steering environment in order to allow both fast interactive simulations for many different scenarios and large HPC simulations for selected scenarios in a hierarchical manner. First tests measuring the performance of the parallel visualisation tools and the simula- 
tion codes on high-performance graphics hardware and HPC architectures, resp., show promising results.

In the future, the combination of the presented tools shall be applied to further scenarios and, accordingly, enhanced with more functionality. In particular, the domain decomposition approach of iFluids will be improved and particle simulation methods will be implemented in iFluids and enhanced in Peano.

Acknowledgements Parts of this work have been carried out with the financial support of KONWIHR - the Kompetenznetzwerk für Technisch-Wissenschaftliches Hoch- und Höchstleistungsrechnen in Bayern.

\section{References}

1. ASHRAE. Standard 55: Thermal Environmental Conditions for Human Occupancy. American Society of Heating, Refrigerating and Air-Conditioning Engineers, Atlanta, 2004.

2. A. Atanasov. Design and implementation of a computational steering framework for CFD simulations. Diploma Thesis, Institut für Informatik, Technische Universität München, 2009.

3. M. Brenk, H.-J. Bungartz, M. Mehl, I. L. Muntean, T. Neckel, and T. Weinzierl. Numerical simulation of particle transport in a drift ratchet. SIAM Journal of Scientific Computing, 30(6):2777-2798, 2008

4. H.-J. Bungartz, M. Mehl, T. Neckel, and T. Weinzierl. The pde framewirk peano applied to computational fluid dynamics. Computational Mechanics, 2009. accepted.

5. D. Fiala. Dynamic Simulation of Human Heat Transfer and Thermal Comfort. Band 41, De Monfort University Leicester, HFT Stuttgart, 1998.

6. A. Gagge. Rational temp. indices of man's thermal env. and their use with a 2-node model of his temp. reg. Fed. Proc., 32:1572-1582, 1973.

7. Sandia National Laboratories. Vtk - visualization toolkit.

8. R.-P. Mundani, A. Düster, J. Knežević, A. Niggl, and E. Rank. Dynamic load balancing strategies for hierarchical p-FEM solvers. In 16th EuroPVM/MPI Conf., pages 305-312, 2009.

9. T. Neckel. The PDE Framework Peano: An Environment for Efficient Flow Simulations. Verlag Dr. Hut, 2009.

10. W. Schroeder, K. Nartin, and B. Lorenson. Visualisation Toolkit: An Object-Oriented Approach to 3D Graphics. Kitware, 2006.

11. J.A.J. Stolwijk. A mathematical model of physiological temperature regulation in man. Contractor report NASA CR-1855, National Aeronautics and Space Administration, Washington D.C., 1971

12. B.A. Szabó, A. Düster, and E. Rank. Encyclopedia of Computational Mechanics, chapter The $p$-version of the Finite Element Method, pages 119-139. John Wiley \& Sons, 2004.

13. C. van Treeck, J. Frisch, M. Egger, and E. Rank. Model-adaptive analysis of indoor thermal comfort. In Building Simulation 2009, Glasgow, Scotland, 2009.

14. C. van Treeck, J. Frisch, M. Pfaffinger, E. Rank, S. Paulke, I. Schweinfurth, R. Schwab, R. Hellwig, and A. Holm. Integrated thermal comfort analysis using a parametric manikin model for interactive real-time simulation. J Building Performance Simulation, in press, 2009.

15. C. van Treeck, P. Wenisch, A. Borrmann, M. Pfaffinger, O. Wenisch, and E. Rank. ComfSim - Interaktive Simulation des thermischen Komforts in Innenräumen auf Höchstleistungsrechnern. Bauphysik, 29(1):2-7, 2007. DOI: 10.1002/bapi.200710000.

16. T. Weinzierl. A Framework for Parallel PDE Solvers on Multiscale Adaptive Cartesian Grids. Verlag Dr. Hut, 2009. 